\title{
Analytic Calculation Method of Zoom Loci for Zoom Lens System with Infinite Object Distance
}

\author{
Jeong Hyo $\mathrm{Oh}^{1}$, Jae Myung $\mathrm{Ryu}^{2}$, and Jae Heung $\mathrm{Jo}^{3 \dagger}$ \\ ${ }^{1}$ Department of Physics, Graduate School, Hannam University, Daejeon 306-791, Korea \\ ${ }^{2}$ Department of Optical Engineering, Kumoh National Institute of Technology, Kumi 730-701, Korea \\ ${ }^{3}$ Department of Optics and Sensors/Department of Physics(Graduate School), Hannam University, Daejeon 306-791, Korea
}

(Received April 11, 2013; Revised manuscript April 26, 2013; Accepted April 26, 2013)

\begin{abstract}
In case of the optical camera system with an infinite object distance, optical designs different from previous systems are required to speed up the auto-focus. As the number of lens groups is increased due to this, the conventional analytic method found it difficult to calculate the locus, and even the one-step advanced calculation method also had the trouble of taking a lot of time. In this paper, we suggested an analytic method for calculating the zoom loci by analyzing movement of one or two groups for situations corresponding to the given back focal length and effective focal length after taking a spline interpolation for each lens group. With this method, we would not only calculate the analytic zoom loci without iterations in every optical system without placing a limit on the group number at the zoom lens systems with the infinite object distance, but we would also show the utilities of this method through many examples.
\end{abstract}

Keywords: Lens design, Zoom locus, Zoom lens design, Spline interpolation

OCIS codes: (110.0110) Imaging systems; (220.1010) Aberrations (global); (220.2740) Geometric optical design; (220.4830) Systems design

\section{무한물점용 줌 렌즈 광학계의 줌 궤적에 대한 해석적 계산법}

$$
\text { 오정효 }{ }^{1} \cdot \text { 류재명 }^{2} \cdot \text { 조재흥 }^{\dagger}
$$

1한남대학교 대학원 물리학과

(우 306-791 대전광역시 대덕구 한남로 70 (오정동)

${ }^{2}$ 국립금오공과대학교 광시스템공학과

(우 730-701 경북 구미시 대학로 61 (양호동)

3한남대학교 공과대학 광·센서공학과/대학원 물리학과

(우 306-791 대전광역시 대덕구 한남로 70 (오정동)

(2013년 4월 11일 받음, 2013년 4월 26일 수정본 받음, 2013년 4월 26일 게재 확정)

무한물점으로 취급하는 카메라 광학계의 경우, 자동초점(auto-focus) 속도를 빠르게 하기 위해 기존 광학계와 다른 형태로 광학 설계를 하게 된다. 이 때문에 군의 개수가 늘어나므로, 기존의 해석적인 방법으로는 궤적 계산이 어렵고, 또한 수치해석적인 방법 은 시간이 많이 걸리는 단점이 있다. 본 논문에서는 각 군을 스플라인 보간을 한 뒤, 주어진 뒤정점초점거리와 유효초점거리를 만족하도록 1 개 또는 2 개 군의 이동량을 해석적으로 구해 줌 궤적을 계산하는 해석적인 방법을 제안하고 이를 유도한다. 이 방법 은 무한물점용 줌 렌즈 광학계에서 군수의 제한 없이 모든 광학계에 대해 반복적인 계산없이 해석적인 궤적을 구할 수 있으며, 다양한 예제를 통하여 그 효용성을 보였다.

Keywords: 렌즈설계, 줌궤적, 줌렌즈설계, 스플라인 보간법

OCIS codes: (110.0110) Imaging systems; (220.1010) Aberrations (global); (220.2740) Geometric optical design; (220.4830) Systems design

\footnotetext{
${ }^{\dagger}$ E-mail: jhjo@hnu.kr

Color versions of one or more of the figures in this paper are available online.
} 


\section{I. 서 론}

과거 기계 가공 기술이 발전하지 못한 때에는 캠(CAM) 부 품의 가공이 쉽지 않기 때문에 이동군과 전체 군 개수를 최 소화하여 줌 광학계를 설계했다. 따라서 이렇게 군의 개수가 적고, 고정군이 많아 구속 조건이 명확하게 주어지는 경우에 는 초기해 없이 해석적으로 줌 궤적을 구할 수 있다. ${ }^{[1]}$ 그러 나 최근에는 기계 가공 기술이 많이 발전해서 캠 부품의 제 작 난이도 및 비용이 많이 낮아졌기 때문에 이러한 군의 개 수에 대한 제한은 많이 완화되었다. 그럼에도 불구하고 6 군 광학계인 JP1999-258504 ${ }^{[2]}$ 의 실시예 3과 같은 아주 군수가 많은 광학계의 경우에는 참고문헌 1 의 해석적인 방법으로는 해를 구하는 것이 매우 어렵고 복잡하다.

또한 최근 국내 카메라 회사를 비롯한 일본 카메라 회사에 서도 기존 일안반사식(SLR, Single Lens Reflex) 카메라에서 미러박스(mirror-box) ${ }^{[3]}$ 가 없는 컴팩트 시스템 카메라(CSC, Compact System Camera) ${ }^{[4]}$ 가 출시되고 있다. CSC의 경우에 는 SLR 카메라와 달리 미러박스가 없기 때문에 카메라를 얇 고 가볍게 만들 수 있다. 그러나 SLR 카메라에 비해 아직 $\mathrm{AF}$ (auto-focus) 속도가 늦은 단점이 있다. 따라서 이를 극복 하기 위해서는 $\mathrm{AF}$ 군의 무게를 줄여서 구동 속도를 높여야 하며, 이를 위해서는 광학계의 형식이 기존과 많이 달라져야 한다. 이러한 예는 SLR용 교환렌즈 제품인 55-200 mm의 망 원렌즈의 제품으로 추정되는 특허 JP2007-206452의 실시예 $1^{[5]}$ 과 50-200 mm 망원렌즈의 제품으로 추정되는 특허 $\mathrm{KR}$ 2011-074291 실시예 $4^{[6]}$ 로부터 알 수 있다. 이는 $\mathrm{AF}$ 군을 경 량화하면서 속도를 높이기 위함이며, 이 때문에 두 광학계의 형태는 군수가 많아지면서 서로 형태가 크게 다르다.

앞서 예를 든 경우들처럼 최근 카메라 광학계는 줌 렌즈의 군수가 많아지는 새로운 형태를 가지기 때문에 참고문헌 1 과 같은 방법으로는 줌 궤적을 구할 수 없다. 이를 해결하기 위해서 최근에는 수치해석적으로 줌 궤적을 구하는 방법 ${ }^{[7,8]}$ 을 사용하였다. 그러나 이 방법은 초기 조건을 보간법으로 결정하고, 주밍 방정식과 구속조건을 연립해서 미분 행렬을 구해서 반복 계산을 하는 것이다. 따라서 이 방법은 반복 계 산 과정에서 많은 시간이 소요된다. 그리고 광학 설계 소프 트웨어(software, $\mathrm{S} / \mathrm{W}$ ) 내에서 궤적을 직접 확인하기 위해서 광학 설계 $\mathrm{S} / \mathrm{W}$ 내부에서 지원하는 프로그래밍 언어로 이러 한 궤적을 계산하게 된다. 최근 광학설계 $\mathrm{S} / \mathrm{W}$ 는 여러 $\mathrm{S} / \mathrm{W}$ 와 데이터 교환을 하기 위해서 API(Application Programming Interface)를 지원하는데, 이 때 광학설계 $\mathrm{S} / \mathrm{W}$ 내부 언어로 궤적 계산을 하면서 계산시간이 많이 걸리게 되고, 이로 인 하여 광학설계 $\mathrm{S} / \mathrm{W}$ 와 데이터 교환을 하는 외부 $\mathrm{S} / \mathrm{W}$ 사이에 런타임(run-time) 오류가 발생하는 경우가 종종 있다.

본 논문에서는 이를 해결하기 위하여 반복 계산을 하지 않 고 해석적으로 무한물점용 줌궤적의 해를 구할 수 있는 새로 운 방법을 제안하여 계산 속도를 획기적으로 개선하고자 한 다. 즉 무한물점용 카메라 줌 렌즈 광학계에서 군의 개수와 구속조건 등의 광학계의 형식에 관계없이 해석적으로 줌 궤
적을 구하는 이론을 유도하고, 여러 가지 특허의 실제 예들 을 가지고 이 방법에 대한 편리성과 정확성을 보여주고자 한다.

\section{II. 궤적 계산 방법}

그림 1 은 무한물점을 갖는 $\mathrm{N}$ 군 줌 광학계의 축상광선에 대한 광로도이다. 줌 궤적은 화각에 관계없이 축상광선이 근 축상점에서의 상고가 항상 0 이 되도록 각 군 사이의 거리를 계산하여 구한다. 이 때 축상광선이 근축상점에서의 상고가 0 이 되면서 주어진 초점거리를 만족해야 한다. 즉 이러한 궤 적을 계산할 때는 상면에서의 축상광선의 상고가 0 이면서 광학계의 전체 초점거리가 원하는 값을 갖는 군간 간격을 구 하는 것이 줌 궤적 계산법이다. 따라서 이를 계산하기 위해 서는 사실상 축상광선에 대한 근축광선추적을 해야 한다. 그 러나 3 군 이상의 줌 렌즈 광학계에서는 이 계산식이 매우 복 잡하여서 손으로 일일이 구하는 것은 매우 비효율적이다. 따 라서 일반적으로 컴퓨터의 프로그래밍 언어로 이 줌 궤적식 을 구현하여 자동으로 구한다. 이 경우 가우스 괄호법 ${ }^{[1,7,8]}$ 을 통해 근축광선추적을 한다면 프로그램밍에 상당히 편리 하다. 광학계의 전체 초점거리와 상면에서의 상고에 관한 식 은 아래와 같이 식 (1)로 표현된다. ${ }^{[1]}$

$$
\begin{aligned}
& {\left[k_{1},-z_{1}, k_{2},-z_{2}, k_{3}, \cdots, k_{N-1},-z_{N-1}, k_{N}\right]=K} \\
& {\left[k_{1},-z_{1}, k_{2},-z_{2}, k_{3}, \cdots, k_{N-1},-z_{N-1}, k_{N},-z_{N}\right]=0}
\end{aligned}
$$

여기서 $k$ 는 각 군의 굴절능, $z$ 는 각 군간 주요점 사이의 거 리, $K$ 는 광학계 전체의 굴절능을 의미한다. 또한 $k$ 와 $z$ 에 사 용된 첨자는 군의 번호를 의미한다.

식 (1)을 zooming 방정식이라고 하며, 모든 광학계의 줌 궤 적은 이 식들로부터 구할 수 있다. 일반적으로 줌 궤적은 설 계가 완료된 상태에서 캠 부품을 설계 및 가공하기 위해 광 학 설계자가 기구 설계자에게 넘겨주는 데이터이다. 따라서 각 군의 굴절능은 줌 궤적을 계산하기에 앞서서 이미 결정이 된다. N군 광학계의 미지수는 각 군 사이의 거리와 초점거리 또는 배율이 되므로, 미지수의 개수는 무한 광학계의 경우 $\mathrm{N}+1$ 개가 된다. 그러나 앞서 서론에서 설명한 바와 같이 광 학계가 복잡해지면 구속조건이 명확히 주어지지 않기 때문 에, 주어진 2 개의 zooming 방정식을 풀 수 있도록 2 개의 변

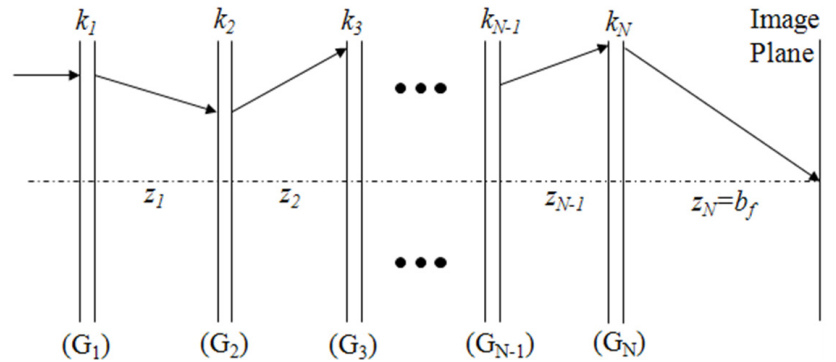

FIG. 1. The optical layout of N-group system. 


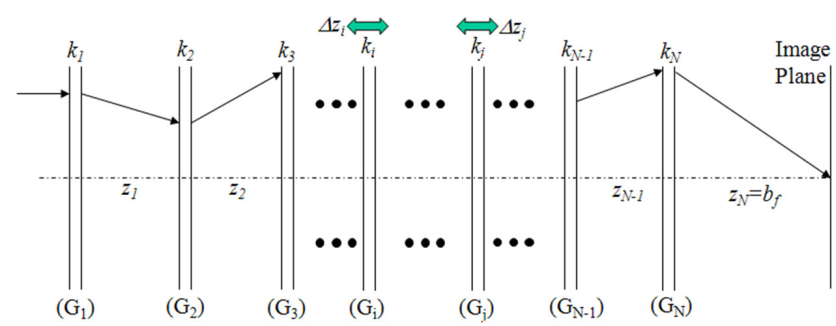

FIG. 2. Optical layout for moving the $\mathrm{i}$-th and $\mathrm{j}$-th group at the lens system with $\mathrm{N}$ groups.

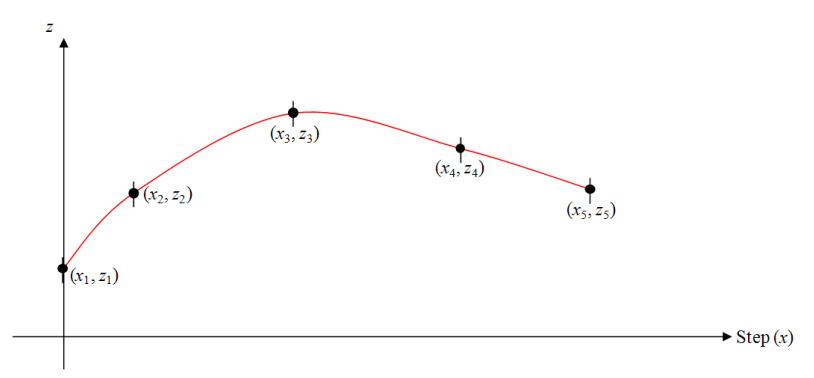

FIG. 3. Example of a locus for a lens group.

수만 미지수로 둘 수 있다.

그림 2는 무한물점에 대해서 주어진 줌 광학계의 초점거리 와 근축 상면에서의 상고가 0 이 되도록 $i$ 번째 및 $j$ 번째 군을 각각 $\Delta z_{i}$ 및 $\Delta z_{j}$ 만큼 움직였을 때의 광로도이다. $\mathrm{N}$ 군 줌 광 학계에서 $i$ 번째 및 $j$ 번째 군을 각각 $\Delta z_{i}$ 및 $\Delta z_{j}$ 만큼 움직였다 고 하면, 식 (1)은 각각 식 (2)와 같이 쓸 수 있다.

$$
\begin{aligned}
& \left.\mid k_{1},-z_{1}, \cdots,-z_{i-1}+\Delta z_{i}, k_{i},-z_{i}-\Delta z_{i}, \cdots,-z_{j-1}+\Delta z_{j}, k_{j},-z_{j}-\Delta z_{j}, \cdots, k_{N-1},-z_{N-1}, k_{N}\right\rfloor=K \\
& {\left[k_{1},-z_{1}, \cdots,-z_{i-1}+\Delta z_{i}, k_{i},-z_{i}-\Delta z_{i}, \cdots,-z_{j-1}+\Delta z_{j}, k_{j},-z_{j}-\Delta z_{j}, \cdots, k_{N-1},-z_{N-1}, k_{N},-z_{N}\right]=0}
\end{aligned}
$$

여기서 광학계 전체 굴절능인 $K$ 를 포함해서 각 군간 거리 $z_{I} \sim z_{N}$ 도 사실상 미지수이다.

그러나 일반적으로 줌 광학계를 설계할 때는 광각단과 망 원단, 그 사이의 1 개 이상의 중간단을 포함하여 설계를 한 다. 보통 광각단과 망원단의 양 끝을 포함해서 줌 배율 3 배 이하는 3 개의 줌 위치를 잡아서, 5 배 이상이면 보통 5 개의
줌 위치를 잡아서 설계를 한다. 따라서 그 중간 위치는 광학 설계에서 이미 결정되는 값이고, 궤적을 구해야 하는 지점의 값들은 보간법을 이용해서 구하면 된다. 이 때 사용하는 보 간법은 여러 가지가 있지만, 각 군의 궤적이 불연속적이거나, 지나치게 급격한 변곡점이 없도록 최대한 완만하고 부드럽 게 보간이 되는 방법을 사용해야 한다. 이 때 설계 줌 위치 에서 연속이고, 1 차 미분이 연속이 되도록 하는 스플라인 (spline) 보간 ${ }^{[9]}$ 을 사용한다면, 비교적 부드러운 궤적을 얻을 수 있다. 그림 3 은 임의의 줌 광학계의 임의의 군에 대한 궤 적을 나타낸 그림이다. $\mathrm{x}$ 축은 줌 스텝(zoom step)이고, $\mathrm{y}$ 축은 군간 간격이다. 또한 곡선 위의 점은 설계 줌 위치를 나타낸다. 그림 3 에서 점이 5 개이므로 설계 줌 위치는 5 개인 경우를 의 미한다.

그림 3 의 곡선의 각 구간을 3 차 다항식으로 나타내고, 설 계 줌 위치에서 연속이고 1 차 미분이 연속이면 다음과 같은 식 (3)를 만족한다. 여기서 $x_{i}$ 는 줌 스텝이고, $z_{i}$ 는 설계 줌 위 치이며, $z_{i}^{\prime}$ 는 설계 줌 위치에 대한 1차 미분값으로 이는 초 기에 주어지는 값이고, $a, b, c, d$ 가 미지수이다.

$$
\begin{aligned}
& z_{i}=a x_{i}^{3}+b x_{i}^{2}+c x_{i}+d \\
& z_{i}^{\prime}=\frac{d z_{i}}{d x_{i}}=3 a x_{i}^{2}+2 b x_{i}+c \\
& z_{i+1}=a x_{i+1}^{3}+b x_{i+1}^{2}+c x_{i+1}+d \\
& z_{i+1}^{\prime}=\frac{d z_{i+1}}{d x_{i+1}}=3 a x_{i+1}^{2}+2 b x_{i+1}+c
\end{aligned}
$$

식 (3)을 행렬 형태로 나타내면 식 (4)가 되고, 식 (4)의 역 행렬을 구해 다항식의 계수를 계산하면 식 (5)와 같다.

$$
\left[\begin{array}{c}
z_{i} \\
z_{i}^{\prime} \\
z_{i+1} \\
z_{i+1}^{\prime}
\end{array}\right]=\left[\begin{array}{cccc}
x_{i}^{3} & x_{i}^{2} & x_{i} & 1 \\
3 x_{i}^{2} & 2 x_{i} & 1 & 0 \\
x_{i+1}^{3} & x_{i+1}^{2} & x_{i+1} & 1 \\
3 x_{i+1}^{2} & 2 x_{i+1} & 1 & 0
\end{array}\right] \cdot\left[\begin{array}{c}
a \\
b \\
c \\
d
\end{array}\right]
$$

$$
\begin{aligned}
{\left[\begin{array}{l}
a \\
b \\
c \\
d
\end{array}\right] } & =\left[\begin{array}{cccc}
x_{i}^{3} & x_{i}^{2} & x_{i} & 1 \\
3 x_{i}^{2} & 2 x_{i} & 1 & 0 \\
x_{i+1}^{3} & x_{i+1}^{2} & x_{i+1} & 1 \\
3 x_{i+1}^{2} & 2 x_{i+1} & 1 & 0
\end{array}\right]^{-1}\left[\begin{array}{c}
z_{i} \\
z_{i}^{\prime} \\
z_{i+1} \\
z_{i+1}^{\prime}
\end{array}\right] \\
& =\frac{1}{\left(x_{i}-x_{i+1}\right)^{3}}\left[\begin{array}{c}
-x_{i}^{2}\left(z_{i}^{\prime}+2 z_{i+1}^{\prime}\right)+x_{i+1} z_{i}^{\prime}+2 z_{i+1}-x_{i+1} z_{i+1}^{\prime}+x_{i}\left(z_{i}^{\prime}+z_{i+1}^{\prime} z_{i}^{\prime}-3 z_{i+1}+x_{i+1} z_{i+1}^{\prime}\right)+x_{i+1}\left(3 z_{i}+2 x_{i+1} z_{i}^{\prime}-3 z_{i+1}+x_{i+1} z_{i+1}^{\prime}\right) \\
-x_{i+1}^{3} z_{i}^{\prime}+x_{i}^{3} z_{i+1}^{\prime}+x_{i}^{2} x_{i+1}\left(2 z_{i}^{\prime}+z_{i+1}^{\prime}\right)-x_{i} x_{i+1}\left(6 z_{i}+x_{i+1} z_{i}^{\prime}-6 z_{i+1}+2 x_{i+1} z_{i+1}^{\prime}\right) \\
-x_{i+1}^{3} z_{i+1}+x_{i} x_{i+1}^{2}\left(3 z_{i}+x_{i+1} z_{i}^{\prime}\right)+x_{i}^{3}\left(z_{i+1}-x_{i+1} z_{i+1}^{\prime}\right)+x_{i}^{2} x_{i+1}\left\{-3 z_{i+1}+x_{i+1}\left(-z_{i}^{\prime}+z_{i+1}^{\prime}\right)\right\}
\end{array}\right]
\end{aligned}
$$


식 (3) (5)와 같이 스플라인 보간법을 사용하여 줌 위치에 대한 미지수 $a, b, c, d$ 를 구하면, 식 (2)에서 $K, z_{1} \sim z_{N}$ 가 모두 구해진다. 이와 동시에 각 군의 굴절능은 광학 설계에서 결 정된다. 이에 따라서 식 (2)에서 남은 미지수는 $\Delta z_{i}$ 및 $\Delta z_{j} 2$ 개가 되므로 이 식들을 풀면 된다. 이렇게 해서 각 군 사이 의 군 간격을 결정하면, 움직이는 군의 앞뒤 군 사이의 거리 및 광학계의 전장이 변하지 않으므로 군간 거리의 구속조건 은 무조건 만족하게 된다. 모든 줌 경우를 다음과 같은 2 가 지 경우로 간단히 나누어 줌 이동에 대한 해석적 해를 구하 면 된다.

\section{1. 초점거리 또는 배율이 변하면서 한 개의 군이 움직 이는 경우(case 1)}

초점거리 또는 배율이 변하는 경우에는 식 (6)만을 만족하 면 된다. 식 (6)은 스플라인 보간법으로 구한 각 군 간격에 따라 발생한 뒤정점초점거리(back focal length, BFL)의 변화 를 $i$ 번째 군으로 보정하는 것 ${ }^{[10]}$ 과 동일하다.

$$
\left[k_{1},-z_{1}, \cdots,-z_{i-1}+\Delta z_{i}, k_{i},-z_{i}-\Delta z_{i}, \cdots, k_{N},-z_{N}\right]=0
$$

식 (6)에서 $k_{i}$ 를 가우스 괄호 밖으로 내보내, $k_{i}$ 에 대한 1 차 식으로 쓰면 식 (7)와 같다.

$$
\begin{aligned}
& {\left[k_{1},-z_{1}, k_{2},-z_{2}, k_{3}, \cdots, k_{i-1},-z_{i-1}+\Delta z_{i}\right] \cdot k_{i} \cdot\left[-z_{i}-\Delta z_{i}, k_{i+1}, \cdots, k_{N-1},-z_{N-1}, k_{N},-z_{N}\right]} \\
& \quad+\left[k_{1},-z_{1}, k_{2},-z_{2}, k_{3}, \cdots,-z_{i-1}-z_{i}, \cdots, k_{N-1},-z_{N-1}, k_{N},-z_{N}\right]=0
\end{aligned}
$$

식 (7)에서 $\Delta z_{i}$ 를 가우스 괄호 밖으로 내보내서 정리하면 식 (8)과 같이 $\Delta z_{i}$ 에 대한 2차 방정식으로 정리할 수 있다.

$$
\begin{aligned}
& A \cdot C \cdot \Delta z_{i}^{2}+(A \cdot D+B \cdot C) \cdot \Delta z_{i}+E=0 \\
& A \equiv\left[k_{1},-z_{1}, k_{2},-z_{2}, k_{3}, \cdots,-z_{i-2}, k_{i-1}\right] \\
& B \equiv\left[k_{1},-z_{1}, k_{2},-z_{2}, k_{3}, \cdots,-z_{i-2}, k_{i-1},-z_{i-1}\right] \\
& C \equiv-\left[k_{i+1},-z_{i+1}, \cdots, k_{N-1},-z_{N-1}, k_{N},-z_{N}\right] \\
& D \equiv\left[-z_{i}, k_{i+1},-z_{i+1}, \cdots, k_{N-1},-z_{N-1}, k_{N},-z_{N}\right] \\
& E \equiv f_{i} \cdot\left[k_{1},-z_{1}, k_{2},-z_{2}, k_{3}, \cdots, k_{N-1},-z_{N-1}, k_{N},-z_{N}\right]
\end{aligned}
$$

무한물점 광학계에서 1군을 이동하는 경우라면, 식 (8)은 보다 간단해져서 식 (9)와 같이 1차 방정식으로 표현된다.

$$
\Delta z_{i}=-\frac{\left[k_{1},-z_{1}, k_{2},-z_{2}, k_{3}, \cdots, k_{N-1},-z_{N-1}, k_{N},-z_{N}\right]}{k_{1} \cdot\left[k_{2},-z_{2}, k_{3}, \cdots, k_{N-1},-z_{N-1}, k_{N},-z_{N}\right]}
$$

이렇게 해서 구한 $\Delta z_{i}$ 가 줌 궤적이 되며, 이 식 (9)를 식 (2) 에 대입하면 유효초점거리(effective focal length, EFL)를 구 할 수 있다.

\section{2. 두 개의 군이 움직이는 경우(case 2)}

이 경우는 두 개의 군이 모두 $\mathrm{EFL}$ 과 $\mathrm{BFL}$ 의 보정군으로 사용되는 경우이며, 식 (2)를 연립해서 풀면 된다. 이 때 식 (2)은 가우스 괄호의 성질 ${ }^{[1]}$ 을 이용해서 식 (10)로 변형할 수 있다.

$$
\begin{aligned}
& \left.\left[k_{1},-z_{1}, \cdots,-z_{i-1}+\Delta z_{i}, k_{i},-z_{i}-\Delta z_{i}, \cdots,-z_{j-1}+\Delta z_{j}, k_{j}\right]=K \cdot \mid k_{j+1},-z_{j+1}, \cdots k_{N},-z_{N}\right\rfloor \\
& {\left[k_{1},-z_{1}, \cdots,-z_{i-1}+\Delta z_{i}, k_{i},-z_{i}-\Delta z_{i}, \cdots,-z_{j-1}+\Delta z_{j}, k_{j},-z_{j}-\Delta z_{j}\right]=-K \cdot\left[-z_{j+1}, \cdots k_{N},-z_{N}\right]}
\end{aligned}
$$

식 (10)의 두 번째 식에서 $\Delta z_{j}$ 를 가우스 괄호 밖으로 꺼내 고, 식 (10)의 첫 번째 식을 다시 대입하고, 이를 다시 $\Delta z_{j}$ 에 대한 1차식으로 정리하면 식 (11)을 얻을 수 있다. 여기서 $\Delta z_{j}$ 의 계수는 $\Delta z_{i}$ 에 대한 2차식이다.

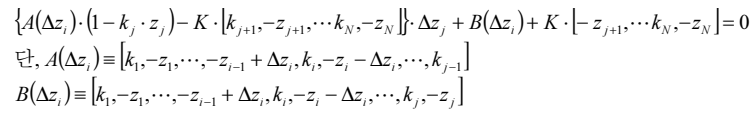

식 (10)의 첫 번째 식을 $\Delta z_{j}$ 에 대한 1차식으로 정리하면 식 (12)을 얻을 수 있다. 여기에서도 $\Delta z_{j}$ 의 계수는 $\Delta z_{i}$ 에 대한 2 차식이다. 식 (12)에 $A\left(\Delta z_{i}\right)$ 는 이미 식 (11)에 정의되어 있다.

$$
\begin{aligned}
& A\left(\Delta z_{i}\right) \cdot k_{j} \cdot \Delta z_{j}+A^{\prime}\left(\Delta z_{i}\right)-K \cdot\left[k_{j+1},-z_{j+1}, \cdots k_{N},-z_{N}\right\rfloor=0 \\
& \text { 단, } A^{\prime}\left(\Delta z_{i}\right) \equiv\left[k_{1},-z_{1}, \cdots,-z_{i-1}+\Delta z_{i}, k_{i},-z_{i}-\Delta z_{i}, \cdots, k_{j}\right]
\end{aligned}
$$

식 (11) 및 식 (12)와 같이 $\Delta z_{j}$ 에 대한 1차 방정식으로 정리 되었으므로, 이를 연립하면 식 (13)과 같이 $\Delta z_{j}$ 가 소거된다.

$$
\begin{aligned}
\Delta z_{j} & =-\frac{B\left(\Delta z_{i}\right)+K \cdot\left[-z_{j+1}, \cdots k_{N},-z_{N}\right\rfloor}{A\left(\Delta z_{i}\right) \cdot\left(1-k_{j} \cdot z_{j}\right)-K \cdot\left[k_{j+1},-z_{j+1}, \cdots k_{N},-z_{N}\right]} \\
& =-\frac{A^{\prime}\left(\Delta z_{i}\right)-K \cdot\left[k_{j+1},-z_{j+1}, \cdots k_{N},-z_{N}\right\rfloor}{A\left(\Delta z_{i}\right) \cdot k_{j}}
\end{aligned}
$$

$\Delta z_{j}$ 가 소거된 식 (13)을 $\Delta z_{i}$ 에 대해 풀기 위해, $A\left(\Delta z_{i}\right), B(\Delta$ $\left.z_{i}\right), A^{\prime}\left(\Delta z_{i}\right)$ 를 $\Delta z_{i}$ 에 대해 전개하면 식 (14)와 같이 $\Delta z_{i}$ 에 관한 2차식을 얻을 수 있다.

$$
\begin{aligned}
& A\left(\Delta z_{i}\right) \equiv\left[k_{1},-z_{1}, \cdots,-z_{i-1}+\Delta z_{i}, k_{i},-z_{i}-\Delta z_{i}, \cdots, k_{j-1}\right]=a_{11} \cdot\left(\Delta z_{i}\right)^{2}+a_{12} \cdot \Delta z_{i}+a_{13} \\
& B\left(\Delta z_{i}\right) \equiv\left[k_{1},-z_{1}, \cdots,-z_{i-1}+\Delta z_{i}, k_{i},-z_{i}-\Delta z_{i}, \cdots, k_{j},-z_{j}\right]=b_{11} \cdot\left(\Delta z_{i}\right)^{2}+b_{12} \cdot \Delta z_{i}+b_{13} \\
& A^{\prime}\left(\Delta z_{i}\right) \equiv\left[k_{1},-z_{1}, \cdots,-z_{i-1}+\Delta z_{i}, k_{i},-z_{i}-\Delta z_{i}, \cdots, k_{j}\right]=a_{21} \cdot\left(\Delta z_{i}\right)^{2}+a_{22} \cdot \Delta z_{i}+a_{23} \\
& \text { 단, } a_{11} \equiv k_{i} \cdot A_{i 1} \cdot C_{i 1} \\
& a_{12} \equiv k_{i} \cdot\left(A_{i 1} \cdot D_{i 1}+B_{i 1} \cdot C_{i 1}\right) \\
& b_{11} \equiv k_{i} \cdot A_{i 1} \cdot C_{i 2} \\
& b_{12} \equiv k_{i} \cdot\left(A_{i 1} \cdot D_{i 2}+B_{i 1} \cdot C_{i 2}\right) \\
& a_{21} \equiv k_{i} \cdot A_{i 1} \cdot C_{i 3} \\
& a_{22} \equiv k_{i} \cdot\left(A_{i 1} \cdot D_{i 3}+B_{i 1} \cdot C_{i 3}\right) \\
& A_{i 1} \equiv\left[k_{1},-z_{1}, \cdots, k_{i-1}\right] \\
& B_{i 1} \equiv\left[k_{1},-z_{1}, \cdots,-z_{i-1}\right]
\end{aligned}
$$




$$
\begin{aligned}
& C_{i 1} \equiv-\left[k_{i+1}, \cdots, k_{j-1}\right] \\
& D_{i 1} \equiv\left[-z_{i}, \cdots, k_{j-1}\right] \\
& a_{13} \equiv E_{i 1} \equiv\left[k_{1},-z_{1}, \cdots, k_{j-1}\right] \\
& C_{i 2} \equiv-\left[k_{i+1}, \cdots, k_{j},-z_{j}\right] \\
& D_{i 2} \equiv\left[-z_{i}, k_{i+1}, \cdots, k_{j},-z_{j}\right] \\
& b_{13} \equiv E_{i 2} \equiv\left[k_{1},-z_{1}, \cdots, k_{j},-z_{j}\right] \\
& C_{i 3} \equiv-\left[k_{i+1}, \cdots, k_{j}\right] \\
& D_{i 3} \equiv\left[-z_{i}, k_{i+1}, \cdots, k_{j}\right] \\
& a_{23} \equiv E_{i 3} \equiv\left[k_{1},-z_{1}, \cdots, k_{j}\right] \\
& c_{1} \equiv K \cdot\left[k_{j+1},-z_{j+1}, \cdots k_{N},-z_{N}\right] \\
& c_{2} \equiv K \cdot\left[-z_{j+1}, \cdots k_{N},-z_{N}\right]
\end{aligned}
$$

$$
\begin{gathered}
\left\{\left(a_{11} \cdot\left(\Delta z_{i}\right)^{2}+a_{12} \cdot \Delta z_{i}+a_{13}\right) \cdot\left(f_{i}-z_{j}\right)-f_{i} \cdot c_{1}\right\} \cdot\left\{c_{1}-a_{21} \cdot\left(\Delta z_{i}\right)^{2}-a_{22} \cdot \Delta z_{i}-a_{23}\right\}+ \\
\left\{a_{11} \cdot\left(\Delta z_{i}\right)^{2}+a_{12} \cdot \Delta z_{i}+a_{13}\right\} \cdot\left\{b_{11} \cdot\left(\Delta z_{i}\right)^{2}+b_{12} \cdot \Delta z_{i}+b_{13}+c_{2}\right\}=0
\end{gathered}
$$

식 (15)에서 $\Delta z_{i}$ 에 대해 정리하면, 식 (16)을 얻을 수 있으 며, 식 (16)에 사용된 $\Delta z_{i}$ 에 대한 방정식의 각 계수에 사용된 $a_{11}, a_{12}, a_{13}, b_{11}, b_{12}, b_{13}, a_{21}, a_{22}, a_{23}$ 등의 정의는 이미 식 (14)에서 정의되었다.

$$
\begin{aligned}
& A_{4} \cdot\left(\Delta z_{i}\right)^{4}+A_{3} \cdot\left(\Delta z_{i}\right)^{3}+A_{2} \cdot\left(\Delta z_{i}\right)^{2}+A_{1} \cdot \Delta z_{i}+A_{0}=0 \\
& \text { 단, } A_{4} \equiv a_{11} \cdot b_{11}-a_{11} \cdot\left(f_{i}-z_{j}\right) \cdot a_{21} \\
& A_{3} \equiv a_{11} \cdot b_{12}+a_{12} \cdot b_{11}-a_{12} \cdot\left(f_{i}-z_{j}\right) \cdot a_{21}-a_{11} \cdot\left(f_{i}-z_{j}\right) \cdot a_{22} \\
& A_{2} \equiv a_{11} \cdot\left(f_{i}-z_{j}\right) \cdot\left(c_{1}-a_{23}\right)-a_{12} \cdot\left(f_{i}-z_{j}\right) \cdot a_{22}+\left\{a_{13} \cdot\left(f_{i}-z_{j}\right)-f_{i} \cdot c_{1}\right\} \cdot a_{21} \\
& \quad+a_{11} \cdot\left(b_{13}+c_{2}\right)+a_{12} \cdot b_{12}+a_{13} \cdot b_{11} \\
& A_{1} \equiv a_{12} \cdot\left(f_{i}-z_{j}\right) \cdot\left(c_{1}-a_{23}\right)-a_{13} \cdot\left(f_{i}-z_{j}\right) \cdot a_{22}+f_{i} \cdot c_{1} \cdot a_{22}+a_{12} \cdot\left(b_{13}+c_{2}\right)+a_{13} \cdot b_{12} \\
& A_{0} \equiv\left\{a_{13} \cdot\left(f_{i}-z_{j}\right)-f_{i} \cdot c_{1}\right\} \cdot\left(c_{1}-a_{23}\right)+a_{13} \cdot\left(b_{13}+c_{2}\right)
\end{aligned}
$$

얻을 수 있으며, 식 (13)의 좌변과 우변의 분모와 분자가 모 두 $\Delta z_{i}$ 에 대한 2차식이므로, 식 (13)을 전개하면 결국 $\Delta z_{i}$ 에 대 한 4차 방정식이 된다는 것을 쉽게 예상할 수 있다. 식 (13) 에 식 (14)를 대입하여, $\Delta z_{i}$ 에 대해 최종적으로 정리하면 식 (15)를 얻을 수 있다.

이 식 (16)에서 구한 $\Delta z_{i}$ 와 식 (13)의 $\Delta z_{j}$ 로부터 case 2의 줌 렌즈 광학계에 대한 줌 궤적을 체계적으로 알 수가 있다.

TABLE 1. The specifications of the third example in JP1999-258504 (Unit: mm)

\begin{tabular}{c|c|c|c|c|c|c}
\hline \hline & \multirow{2}{*}{ EFL } & \multirow{2}{*}{ H1 } & \multirow{2}{*}{ H2 } & \multicolumn{3}{|c}{ Thickness } \\
\cline { 4 - 7 } & & & & Wide & Normal & Tele \\
\hline 1st group & 136.247314 & 5.118750 & 9.283673 & 3.58226 & 41.34888 & 61.41823 \\
\hline 2nd group & -39.006352 & 7.953922 & 6.809153 & 29.97808 & 16.40337 & 3.49288 \\
\hline 3rd group & 62.468148 & 1.364312 & 7.123414 & 4.41870 & 17.99341 & 30.90390 \\
\hline 4th group & -230.818116 & -1.584365 & 3.299259 & 36.06116 & 23.86123 & 30.96297 \\
\hline 5th group & 60.406382 & 4.217601 & 2.630522 & 27.13692 & 17.81480 & 1.87224 \\
\hline 6th group & -50.643613 & 1.444391 & 1.786775 & 40.70027 & 62.22233 & 71.063162 \\
\hline
\end{tabular}

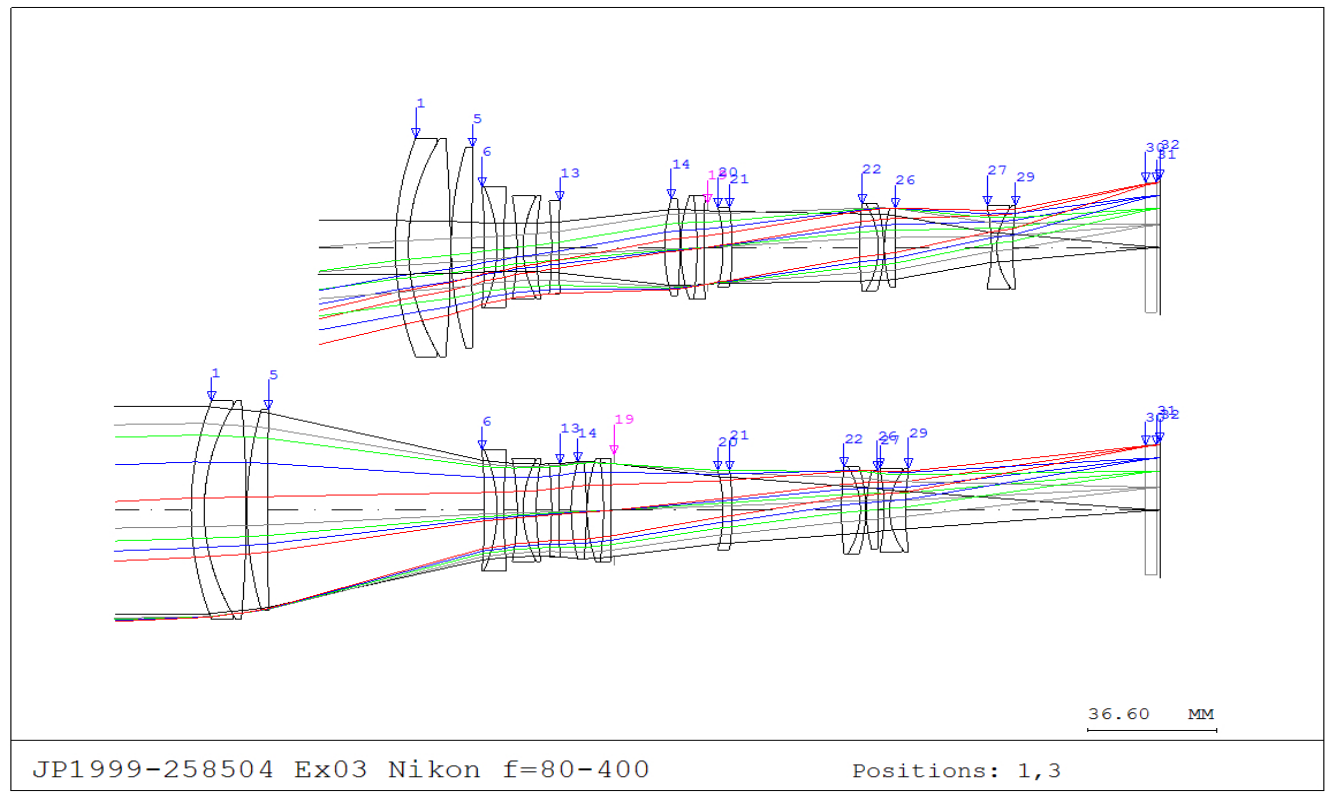

FIG. 4. Optical layout of the third example in JP1999-258504. ${ }^{[2]}$ 
그리고 식 (16)의 4개의 근들 중 물리적으로 타당하고 기계 공학적으로 제작이 가능하며, 가장 이동거리가 작은 해를 선 택하여 실제로 적용하면 된다.

\section{III. 해석적 줌 궤적 계산법을 사용한 궤적 계산의 예}

무한물점용 줌 렌즈 광학계의 해석적 줌 궤적 계산법을 사 용하여 실제의 줌 궤적을 계산하는 실 예를 보여주고자 한다. 표 1은 JP1999-258504 $4^{[2]}$ 의 실시예 3에서 줌 궤적 계산에 필 요한 물리량을 나타낸 것으로서, EFL은 d-line에서 군별 초 점거리, $\mathrm{H} 1$ 은 물체측 첫 번째 면에서 제 1 주요면까지의 거리, $\mathrm{H} 2$ 는 제 2 주요면에서 상측 마지막 면까지의 거리이고, Thickness
는 군간 간격으로, 광각단(wide), 중간단(normal), 망원단(tele) 별로 값이 달라지는 것을 알 수 있다.

그림 4는 표 1에서 보여준 SLR용 교환렌즈 제품인 80-400 mm F/4.5 5.6 망원렌즈의 제품으로 추정되는 JP1999-258504 ${ }^{[2]}$ 의 실시예 3 에 대한 광로도이다. 이 광학계는 2 군과 4 군이 고정 군이고, 1 군과 조리개가 포함된 3 군, 5 군과 6 군이 변배시 이 동되며, $\mathrm{AF}$ 는 가장 마지막 군인 6 군으로 수행한다. 이러한 6 군 광학계의 경우에는 참고문헌 1 의 해석적인 방법으로는 해를 구하는 것이 매우 복잡하다.

2장의 2.1 의 case 1 은 계산이 비교적 간단하므로, 2.2 의 case 2 인 초점거리가 주어진 경우부터 살펴보고자 한다. 2.2 에서 설명한대로 설계하고자 하는 줌 렌즈 광학계가 원하는 $\mathrm{EFL}$ 과 $\mathrm{BFL}$ 을 만족해야 하므로 2 개의 이동군을 선택해야 하

TABLE 2. The locus table of the third example in JP1999-258504 by compensation of the 1st \& 6th groups (Unit: mm)

\begin{tabular}{c|c|c|c|c|c|c|c|c}
\hline \hline Step & EFL & thi s5 & thi s13 & thi s19 & thi s21 & thi s26 & thi s29 & thi s31 \\
\hline 0 & 82.00867 & 3.582261 & 29.978084 & 4.418699 & 36.061159 & 27.136917 & 37.724924 & 1 \\
\hline 100 & 112.97255 & 20.045991 & 25.885465 & 8.511318 & 31.576539 & 24.008164 & 45.338296 & 1 \\
\hline 200 & 143.93644 & 30.184373 & 22.113757 & 12.283026 & 27.975208 & 21.509345 & 51.438444 & 1 \\
\hline 300 & 174.90032 & 37.322488 & 18.66296 & 15.733823 & 25.257167 & 19.310798 & 56.355032 & 1 \\
\hline 400 & 205.8642 & 42.807051 & 15.533073 & 18.86371 & 23.422414 & 17.209327 & 60.291255 & 1 \\
\hline 500 & 236.82809 & 47.273781 & 12.724097 & 21.672686 & 22.47095 & 15.068227 & 63.383818 & 1 \\
\hline 600 & 267.79197 & 51.044885 & 10.236032 & 24.160751 & 22.402775 & 12.797354 & 65.722867 & 1 \\
\hline 700 & 298.75585 & 54.284661 & 8.068877 & 26.327906 & 23.21789 & 10.34602 & 67.359087 & 1 \\
\hline 800 & 329.71973 & 57.072739 & 6.222633 & 28.17415 & 24.916293 & 7.698106 & 68.308598 & 1 \\
\hline 900 & 360.68362 & 59.44485 & 4.6973 & 29.699483 & 27.497985 & 4.864569 & 68.560445 & 1 \\
\hline 1000 & 391.6475 & 61.418227 & 3.492878 & 30.903905 & 30.962966 & 1.872236 & 68.087798 & 1 \\
\hline
\end{tabular}

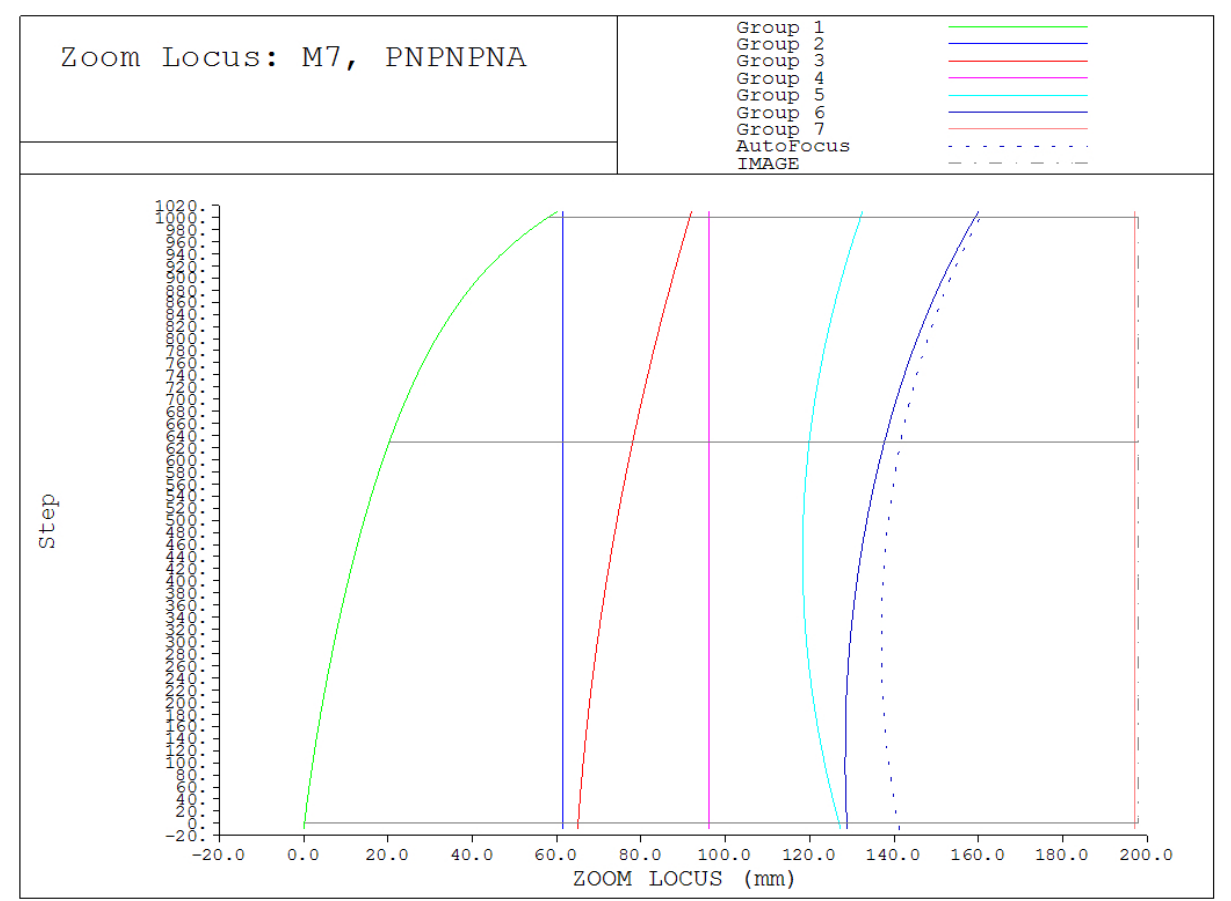

FIG. 5. The zoom locus of the third example in JP1999-258504 by Table 2. 
는데, 고정군인 2 군과 4 군을 제외한 4 개의 군 중에서 2 개를 임의적으로 선택해야 한다. 이 때 경우의 수는 6 가지가 있는 데, 본 논문에서는 여섯 가지 중에서 편의상 1 군과 6 군, 3 군 과 5 군, 1 군과 3 군을 선택한 세 가지 형태만 논하도록 한다.

표 2는 1군과 6군을 선택했을 때, 식 (13)과 식 (16)을 만족 하는 군간 거리를 계산한 결과이다. 줌 궤적을 계산하는 이 유가 각 이동군의 캠을 가공하거나 전기모터 등으로 각 군의 이동을 제어하기 위한 목적이므로, 수백 등분 이상으로 세밀 하게 계산하는 것이 일반적이나 본 연구에서 이 모든 값을 모두 보이는 것은 의미가 없으므로 총 1000 등분 중 줌 양 끝 단을 포함해서 11 등분만 표시했다. 그림 5 는 표 2 의 값을 그 린 것으로 이는 각 군의 궤적이며, 왼쪽 곡선부터 차례대로 1 군 6 군을 의미한다. 표 2 를 그릴 때에는 각 군의 간격을 직
접 그리는 것이 아니고, 궤적을 그리려는 군까지의 누적 군 간 거리를 계산한다. 마찬가지로 표 3 은 3 군과 5 군을 선택했 을 때 식 (13)과 식 (16)을 만족하는 군간 거리를 계산한 결 과이고, 그림 6 은 표 3 과 같은 각 군의 궤적을 나타낸 것이 다. 마지막으로 표 4 는 1 군과 3 군을 선택했을 때의 결과이고, 그림 7은 표 4 와 같은 각 군의 궤적을 나타낸 것이다. 표 2 표 4에서 보는 것처럼, 스플라인 보간된 $\mathrm{EFL}$ 과 $\mathrm{BFL}$ 이 만족 되도록 궤적을 계산했기 때문에 EFL과 BFL이 모두 일치하 는 것을 확인할 수 있다. 그림 5 그림 7에서 표시된 7번째 군은 OLPF(Optical Low Pass Filter) 및 촬상소자의 보호 유 리를 포함한 굴절능이 없는 양면 평면 소자를 의미한다. 따 라서 실제 $\mathrm{BFL}$ 은 'thi $\mathrm{s} 29$ ' 및 'thi $\mathrm{s} 31$ '를 합한 값이 된다. 표 3 표 4에서 'thi s29' 및 'thi s31'를 합한 값이 일정한 것을 알

TABLE 3. The locus table of the third example in JP1999-258504 by compensation of the 3rd \& 5th (Unit: mm)

\begin{tabular}{c|c|c|c|c|c|c|c|c}
\hline \hline Step & EFL & thi s5 & thi s13 & thi s19 & thi s21 & thi s26 & thi s29 & thi s31 \\
\hline 0 & 82.00867 & 3.582261 & 29.978084 & 4.418699 & 36.061159 & 27.136917 & 37.724924 & 1 \\
\hline 100 & 112.972553 & 15.651828 & 22.292615 & 12.104168 & 30.115497 & 26.092563 & 44.714938 & 1 \\
\hline 200 & 143.936436 & 26.324513 & 18.58427 & 15.812513 & 26.363045 & 23.733606 & 50.826346 & 1 \\
\hline 300 & 174.900319 & 35.600315 & 16.90874 & 17.488043 & 24.4148 & 20.449048 & 56.059148 & 1 \\
\hline 400 & 205.864202 & 43.479235 & 16.29642 & 18.100363 & 23.793288 & 16.716363 & 60.413345 & 1 \\
\hline 500 & 236.828085 & 49.961272 & 16.126787 & 18.269996 & 24.110824 & 12.923237 & 63.888935 & 1 \\
\hline 600 & 267.791968 & 55.046428 & 15.869605 & 18.527178 & 25.088269 & 9.348808 & 66.485919 & 1 \\
\hline 700 & 298.75585 & 58.734701 & 15.000708 & 19.396075 & 26.535364 & 6.183334 & 68.204298 & 1 \\
\hline 800 & 329.719733 & 61.026092 & 13.003731 & 21.393052 & 28.28058 & 3.598346 & 69.044071 & 1 \\
\hline 900 & 360.683616 & 61.920601 & 9.37309 & 25.023693 & 30.013065 & 1.904696 & 69.005237 & 1 \\
\hline 1000 & 391.647499 & 61.418227 & 3.492878 & 30.903905 & 30.962966 & 1.872236 & 68.087798 & 1 \\
\hline
\end{tabular}

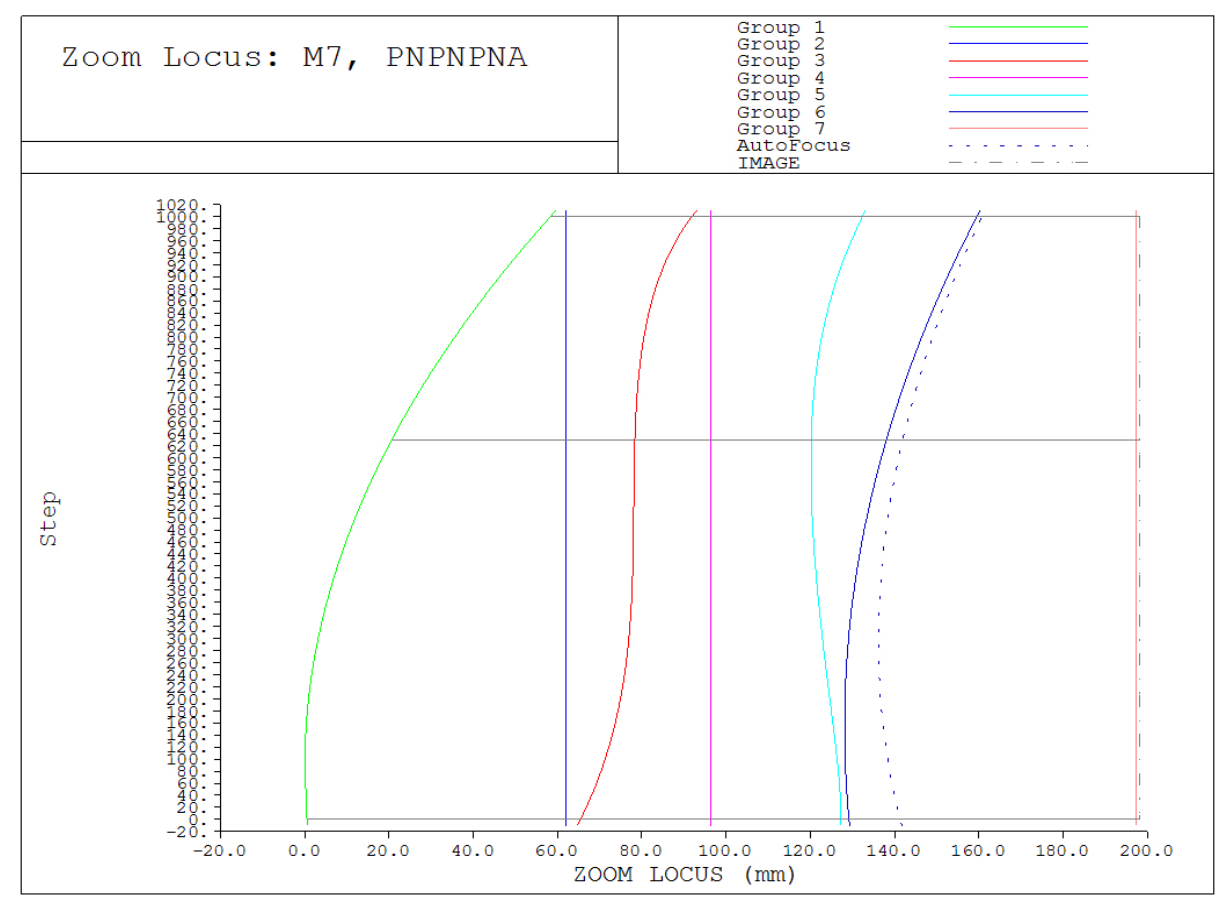

FIG. 6. The zoom locus of the first example in JP1999-258504 by Table 3. 
TABLE 4. The locus table of the third example in JP1999-258504 by compensation of the 1st \& 3th (Unit: mm)

\begin{tabular}{c|c|c|c|c|c|c|c|c}
\hline \hline Step & EFL & thi s5 & thi s13 & thi s19 & thi s21 & thi s26 & thi s29 & thi s31 \\
\hline 0 & 82.00867 & 3.582261 & 29.978084 & 4.418699 & 36.061159 & 27.136917 & 37.724924 & 1 \\
\hline 100 & 112.972553 & 19.103767 & 24.836034 & 9.560749 & 31.576539 & 24.631521 & 44.714938 & 1 \\
\hline 200 & 143.936436 & 29.380272 & 21.125985 & 13.270798 & 27.975208 & 22.121443 & 50.826346 & 1 \\
\hline 300 & 174.900319 & 36.974329 & 18.200727 & 16.196057 & 25.257167 & 19.606681 & 56.059148 & 1 \\
\hline 400 & 205.864202 & 42.938001 & 15.719367 & 18.677416 & 23.422414 & 17.087237 & 60.413345 & 1 \\
\hline 500 & 236.828085 & 47.772947 & 13.483223 & 20.91356 & 22.47095 & 14.563111 & 63.888935 & 1 \\
\hline 600 & 267.791968 & 51.744226 & 11.374083 & 23.0227 & 22.402775 & 12.034301 & 66.485919 & 1 \\
\hline 700 & 298.75585 & 55.005732 & 9.327933 & 25.06885 & 23.21789 & 9.500809 & 68.204298 & 1 \\
\hline 800 & 329.719733 & 57.657557 & 7.322241 & 27.074543 & 24.916293 & 6.962634 & 69.044071 & 1 \\
\hline 900 & 360.683616 & 59.774214 & 5.366801 & 29.029982 & 27.497985 & 4.419777 & 69.005237 & 1 \\
\hline 1000 & 391.647499 & 61.418227 & 3.492878 & 30.903905 & 30.962966 & 1.872236 & 68.087798 & 1 \\
\hline
\end{tabular}

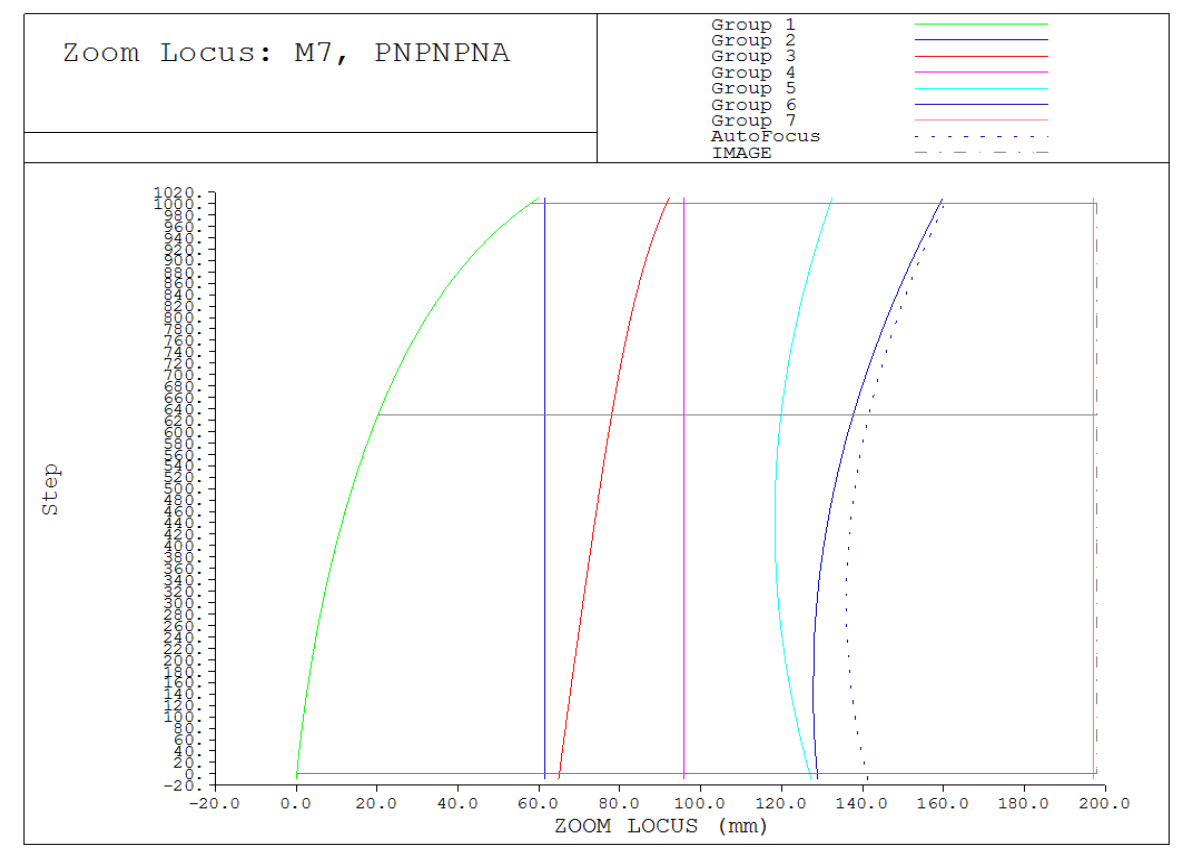

FIG. 7. The zoom locus of the first example in JP1999-258504 by Table 4.

TABLE 5. The locus table of the third example in JP1999-258504 by compensation of the 5th group for linearization of 1st group (Unit: mm)

\begin{tabular}{c|c|c|c|c|c|c|c|c}
\hline \hline Step & EFL & thi s5 & thi s13 & thi s19 & thi s21 & thi s26 & thi s29 & thi s31 \\
\hline 0 & 82.00867 & 3.582261 & 29.978084 & 4.418699 & 36.061159 & 27.136917 & 37.724924 & 1 \\
\hline 100 & 92.002977 & 9.365859 & 28.807108 & 5.589675 & 33.719017 & 25.769441 & 41.43454 & 1 \\
\hline 200 & 103.820535 & 15.149457 & 27.307788 & 7.088995 & 31.438729 & 24.48974 & 44.994528 & 1 \\
\hline 300 & 117.92308 & 20.933055 & 25.480126 & 8.916657 & 29.274887 & 23.243222 & 48.404887 & 1 \\
\hline 400 & 134.909875 & 26.716652 & 23.32412 & 11.072663 & 27.295756 & 21.961623 & 51.665617 & 1 \\
\hline 500 & 155.574829 & 32.500249 & 20.839771 & 13.557012 & 25.594561 & 20.551715 & 54.776719 & 1 \\
\hline 600 & 181.00629 & 38.283845 & 18.027079 & 16.369705 & 24.307598 & 18.877206 & 57.738192 & 1 \\
\hline 700 & 212.792289 & 44.067441 & 14.886043 & 19.51074 & 23.645908 & 16.727052 & 60.550037 & 1 \\
\hline 800 & 253.530551 & 49.851037 & 11.416665 & 22.980118 & 23.958674 & 13.752071 & 63.212252 & 1 \\
\hline 900 & 308.393276 & 55.634632 & 7.618943 & 26.77784 & 25.89171 & 9.306449 & 65.72484 & 1 \\
\hline 1000 & 391.647499 & 61.418227 & 3.492878 & 30.903905 & 30.962966 & 1.872236 & 68.087798 & 1 \\
\hline
\end{tabular}




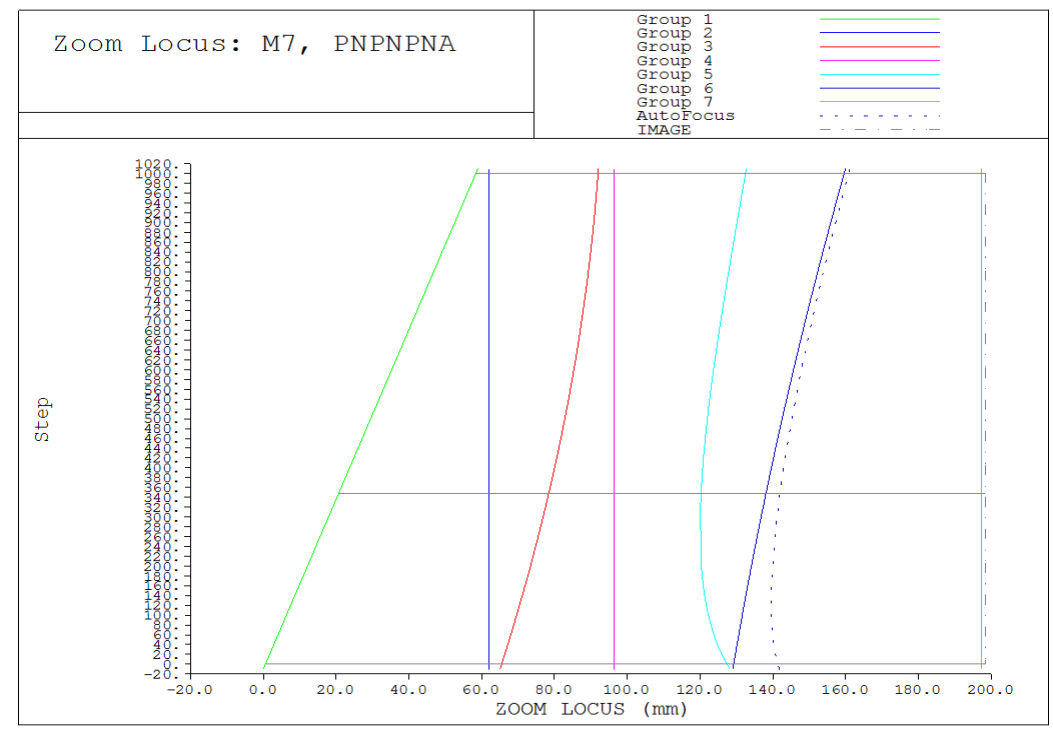

FIG. 8. The zoom locus of the first example in JP1999-258504 by Table 5 .

TABLE 6. The locus table of the third example in JP1999-258504 by compensation of the 1th group for linearization of 3rd group (Unit: mm)

\begin{tabular}{c|c|c|c|c|c|c|c|c}
\hline \hline Step & EFL & thi s5 & thi s13 & thi s19 & thi s21 & thi s26 & thi s29 & thi s31 \\
\hline 0 & 82.00867 & 3.582261 & 29.978084 & 4.418699 & 36.061159 & 27.136917 & 37.724924 & 1 \\
\hline 100 & 94.723813 & 8.621285 & 27.329562 & 7.067221 & 32.097881 & 25.916997 & 42.908121 & 1 \\
\hline 200 & 112.739632 & 16.649577 & 24.681041 & 9.715742 & 28.902038 & 24.406734 & 47.614226 & 1 \\
\hline 300 & 135.48746 & 25.332075 & 22.032519 & 12.364264 & 26.47363 & 22.606126 & 51.843241 & 1 \\
\hline 400 & 162.379856 & 33.445823 & 19.383998 & 15.012785 & 24.812658 & 20.515174 & 55.595164 & 1 \\
\hline 500 & 192.873895 & 40.537587 & 16.735477 & 17.661306 & 23.919121 & 18.133878 & 58.869997 & 1 \\
\hline 600 & 226.536769 & 46.53523 & 14.086957 & 20.309826 & 23.79302 & 15.462237 & 61.667739 & 1 \\
\hline 700 & 263.111359 & 51.513775 & 11.438437 & 22.958346 & 24.434353 & 12.500253 & 63.98839 & 1 \\
\hline 800 & 302.579115 & 55.585437 & 8.789917 & 25.606866 & 25.843122 & 9.247925 & 65.83195 & 1 \\
\hline 900 & 345.217324 & 58.856941 & 6.141397 & 28.255386 & 28.019326 & 5.705253 & 67.19842 & 1 \\
\hline 1000 & 391.647499 & 61.418227 & 3.492878 & 30.903905 & 30.962966 & 1.872236 & 68.087798 & 1 \\
\hline
\end{tabular}

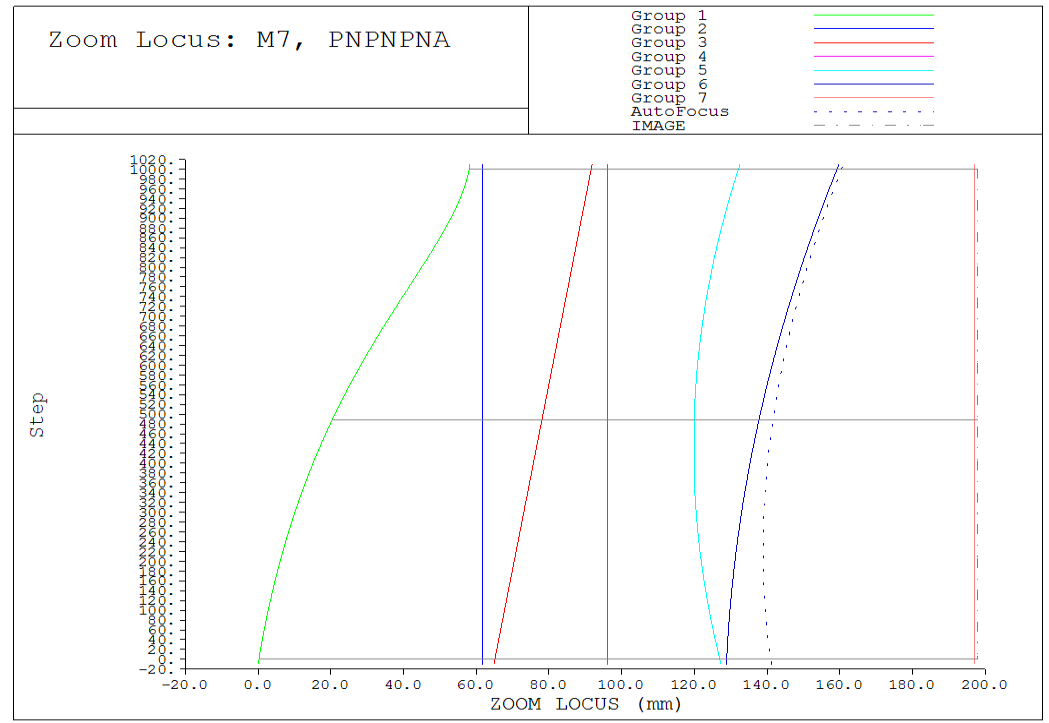

FIG. 9. The zoom locus for the first example in JP1999-258504 by Table 6 . 
수 있다. BFL이 스플라인 보간된 값을 만족하도록 계산했는 데, 표 2의 BFL에 해당하는 값이 표 3 표 4과 다른 이유는 표 2를 계산했을 때에는 마지막 군인 6 군을 보상자로 사용했 기 때문이다. 단, 표 2 표 4에서 고정군인 2군 또는 4군에서 상면까지의 거리는 모두 일정한 것을 확인할 수 있다.

이번에는 2.1 의 case 1 로서 초점거리 또는 배율이 가변이 고, 한 개의 군만이 $\mathrm{BFL}$ 변화를 보상하는 경우에 대한 줌 궤 적을 구하는 것이다. 움직이는 군이 4 개이고 특정군을 선형 으로 궤적을 계산한다면, 1 군과 3 군이 선형이 될 수 있다. 따 라서 경우의 수는 총 6 가지가 되겠으나, 여기서도 실무적으 로 많이 사용될 수 있는 1 군을 선형으로 하고 5 군으로 보정 하는 것과 3 군을 선형으로 하고 1 군으로 보정하는 것만을 계 산한다. 표 5는 1 군이 선형이고 5군으로 보정했을 때, 식 (8) 을 만족하는 군간 거리를 계산한 결과이고, 그림 8 은 표 5 와 같은 각 군의 궤적을 나타낸 것이다. 표 6 은 3 군이 선형이고 1 군으로 보정했을 때, 식 (9)를 만족하는 군간 거리를 계산한 결과이고, 그림 9 는 표 6 과 같은 각 군의 궤적을 나타낸 것 이다.

\section{IV. 결 론}

최근에는 $\mathrm{CAM}$ 가공 기술이 발전하여 이를 적용하는 데 문제가 없고, 카메라 광학계의 경우, $\mathrm{AF}$ 속도를 빠르게 하기 위해 기존 광학계와 다른 형태로 광학 설계를 하게 된다. 이 때문에 군의 개수가 늘어나므로, 기존의 해석적인 방법으로 는 궤적 계산이 어렵고, 또한 수치해석적인 방법은 시간이 많이 걸리는 단점이 있다. 이러한 문제점들을 해결하기 위하 여 본 논문에서는 줌 렌즈 광학계의 각 군을 스플라인 보간 을 한 뒤, 제약조건으로 주어진 $\mathrm{BFL}$ 및 $\mathrm{EFL}$ 을 만족하도록 하는 1 개 또는 2 개 군의 이동량을 해석적으로 구해 궤적을 계산하는 방법에 대해 제안하고 이에 대한 이론식을 해석적 으로 유도하였다. 이러한 방법으로 무한물점용 줌 렌즈 광학 계에서 군의 개수의 제한 없이 모든 광학계에 대해 반복적인 수치해석적 계산과정이 없이 해석적인 방법만을 통하여 줌 궤적을 구할 수 있었다. 모든 줌 렌즈 광학계의 유형은 (1) 무한물점용 줌 렌즈 광학계에 대한 초점거리 또는 배율이 변 하면서 한 개의 군이 움직이는 경우(case 1)와 (2) 무한물점 용 줌 렌즈 광학계에 대하여 두 개의 군이 움직이는 경우 (case 2)에 대하여 해석적인 해를 구하였다.

이 결과의 유용성을 증명하기 위하여 참고문헌 1 의 해석적 인 방법으로는 해를 구하는 것이 매우 어렵고 복잡한 6군 줌 렌즈 광학계인 참고문헌 2인 JP1999-258504의 실시 예들을
사용하였다. 그 결과 case 1 에 대한 줌 궤적 결과인 그림 8과 그림 9 및 case 2 의 줌 궤적에 대한 결과인 그림 5, 그림 6 , 그림 7을 구하고 이를 해석하는 과정을 보듯이 비교적 쉽게 그리고 체계적이면서 통합적인 해석적 궤적 계산법으로부터 다양하게 줌 궤적을 구할 수 있음을 보여 주었다.

이러한 무한물점용 줌 렌즈 광학계에 대한 줌 궤적의 해석 적 해를 이용한 방법은 유한물점으로도 확장이 가능하리라 생각하며, 다중배치 광학계나 매크로 렌즈와 같은 다양한 유 사 줌 렌즈 광학계에도 적용이 가능하리라 생각한다.

\section{References}

1. J. H. Jung, "Paraxial design and locus analysis of zoom lens system," Dissertation of Ph. D. Degree, Kyungnam University (1994).

2. A. Masayuki, "Vibration proof zoom lens," Japan Patent JP1999-258504 (1999).

3. Camera Lens Hyaka (Encyclopedia), 1st ed. (Shashin Kogyo Shoppan Co., Ltd., 2001), Chapter 76.

4. Amateur Photographer, "AP award winners 2011," (IPC Media Ltd., 2011), http://www.amateurphotographer.co.uk/ news/AP Awards 2011 news 305140.html.

5. A. Shibayama and K. Mizuguchi, "Zoom lens with vibrationproof function," Japan Patent JP2006-221092 (2006).

6. S. Yoneyama, "Zoom lens and photographing apparatus having the same," Korean Patent 10-2011-0074291 (2011).

7. N. J. Yoo, W. S. Kim, J. H. Jo, J. M. Ryu, H. J. Lee, and G. M. Kang, "Numerical calculation method for paraxial zoom loci of complicated zoom lenses with infinite object distance by using Gaussian Bracket method," Korean J. Opt. Photon. (Hankook Kwanghak Hoeji) 18, 410-420 (2007).

8. D. K. Lee, N. J. Yoo, J. H. Jo, J. M. Ryu, G. M. Kang, and H. J. Lee, "General numerical calculation method for paraxial zoom loci of zoom lenses with finite object distance by using Gaussian Bracket method," Korean J. Opt. Photon. (Hankook Kwanghak Hoeji) 20, 156-165 (2009).

9. W. H. Press, S. A. Teukolsky, W. T. Vetterling, and B. P. Flannery, Numerical Recipes in $C$, 2nd ed. (Cambridge University Press, 1992), Chapter 3.

10. J. M. Ryu, G. M. Kang, H. J. Lee, H. K. Lee, and J. H. Jo, "Correction method for the variation of the image plane generated by various symmetric error factors of zoom lenses of digital still cameras and estimation method of defect rate due to the correction," Korean J. Opt. Photon. (Hankook Kwanghak Hoeji) 15, 420-429 (2006). 